\title{
Rotation in Globular Cluster stars ${ }^{\star}$
}

\section{Turn-off and subgiant stars in NGC 104, NGC 6397 and NGC 6752}

\author{
S. Lucatello ${ }^{1,2}$ and R. G. Gratton ${ }^{2}$ \\ 1 Dipartimento di Astronomia, Università di Padova, Vicolo dell'Osservatorio, 2, 35122 Padova, Italy \\ 2 INAF - Osservatorio Astronomico di Padova, Vicolo dell'Osservatorio 5, 35122 Padova, Italy \\ e-mail: gratton@pd.astro.it
}

Received 16 July 2001 / Accepted 21 May 2003

\begin{abstract}
We present a derivation of upper limits to the rotation of Main Sequence stars in three globular clusters using spectra obtained during time allocated to the ESO Large Programs 165-L0263 and 167.D-0173, with UVES at VLT2 (Kueyen). The stars analyzed in this work do not show any evidence of high values of rotational velocities as far as the outer layers are concerned, in particular robust estimates for the upper limits of the values of the mean projected rotational velocities are placed, about $\overline{v_{\text {rot }} \sin i}=3.5 \pm 0.2 \mathrm{~km} \mathrm{~s}^{-1}$ and about $\overline{v_{\text {rot }} \sin i}=4.7 \pm 0.2 \mathrm{~km} \mathrm{~s}^{-1}$ for, respectively, the program turn-off and subgiant stars. On the basis of statistical considerations, assuming that inclination of the rotational axis $i$ is randomly oriented, and that all stars within the same group rotate at the same rate, we obtain estimates for the values of the true rotational velocities. These values are upper limits to the true rotational velocities if there is some star-to-star scatter in rotational or macro-turbulent velocities. The mean values of these upper limits for $v_{\text {rot }}$ for the stars of the same spectral type averaged over the three clusters are found to be respectively, $\bar{v}_{\text {rot }} \leq 3.5 \pm 0.4 \mathrm{~km} \mathrm{~s}^{-1}$ and $\bar{v}_{\text {rot }} \leq 2.6 \pm 1.1 \mathrm{~km} \mathrm{~s}^{-1}$. Thus, the explanation for the large rotational velocities found for the Horizontal Branch stars must be looked for either in the rotation of the core not detectable in the outer layers (Sills \& Pinsennault 2000) or in the acquisition of angular momentum during their evolution.
\end{abstract}

Key words. stars: rotation - globular clusters: individual: NGC 104 - globular clusters: individual: NGC 6397 globular clusters: individual: NGC 6752

\section{Introduction}

Since the first detection of fast rotating stars located on the Horizontal Branch of several Globular clusters (Peterson 1983; Behr et al. 2000a,b), rotation of old population stars has started to be debated. In order to understand the mechanism that leads to such large rotational velocities, it is important to have an estimate of such quantity during the main sequence phase. Given the large cluster ages, a low value for the rotational velocity $\left(\leq 2 \mathrm{~km} \mathrm{~s}^{-1}\right)$ is expected by using e.g. the Skumanich law; however no observational result has been obtained so far.

The high resolution spectra of turn-off (TO hereafter) and subgiant (SG hereafter) stars collected primarily for performing abundance analysis of globular cluster stars offered an ideal opportunity to give a direct estimate of the rotational velocities of such stars using the cross-correlation technique (Tonry \& Davis 1979). In fact, the breadth of the peak of the cross-correlation of two stars provides an estimate of the quadratic sum of the global velocity fields within the two stellar

Send offprint requests to: S. Lucatello,

e-mail: lucatello@pd.astro.it

* Based on observations collected at the European Southern Observatory, Chile. atmospheres, i.e. a sum of the contribution due to rotational velocities, macro and micro-turbulent velocities. Using as templates spectra of stars whose atmospheric parameters are very similar to those of the program stars, and modeling the intrinsic Doppler broadening of such templates, it is possible to obtain an estimate of the atmospheric velocity field for the program stars. Accurate determinations of such quantity would require a detailed knowledge of the dynamical behavior of the atmosphere, which is not available yet. However, as will be shown, the application of this method coupled with an appropriate calibration on a set of stars with already studied rotational velocity, provides a reliable measurement of the value of $v_{\text {rot }} \sin i$. Moreover, through statistical consideration of the scatter of individual values compared to expectations for randomly distributed orientation of the rotation axis, even more stringent constraints on the true rotational velocities of the stars observed can be derived.

\section{Observations and data reduction}

The observations were collected using UVES at the VLT, in four separate runs, in June and September 2000, August and October 2001 during the time allocated for the ESO Large 
Table 1. Atmospheric parameters for the template stars.

\begin{tabular}{lcccc}
\hline \hline Star & $\begin{array}{c}T_{\text {eff }} \\
\text { ID }\end{array}$ & $\begin{array}{c}\log g \\
\mathrm{dex}\end{array}$ & $\begin{array}{c}v_{\text {micro }} \\
\mathrm{km} \mathrm{s}^{-1}\end{array}$ & $\begin{array}{c}{[\mathrm{Fe} / \mathrm{H}]} \\
\mathrm{dex}\end{array}$ \\
\hline G 4-37 & 5937 & 3.50 & 1.50 & -2.88 \\
G 126-62 & 6085 & 4.12 & 0.79 & -1.53 \\
G 141-19 & 4997 & 2.25 & 1.30 & -2.96 \\
HD 120559 & 5383 & 4.57 & 0.00 & -0.90 \\
HD 121004 & 5583 & 4.37 & 0.00 & -0.73 \\
\hline
\end{tabular}

Programs 165-L0263 and 167.D-0173, devoted to the abundance analysis of globular cluster dwarfs.

A total of 39 stars in 3 globular cluster were observed, with the same instrumental setting. More precisely, we observed 8 subgiants and 5 turn-off stars in NGC 104-47 Tuc, 9 TO and 9 SG stars in NGC 6752, 5 TO and 3 SG stars in NGC 6397. Two of the SG in NGC 104-47 Tuc have been observed twice, the first time during the August 2001 run, and the other in the October 2001 one.

The location of the program stars in the color-magnitude diagram is shown in Gratton et al. (2001) and Carretta et al. (2003) and the photometric data are taken from Grundahl et al. (2000).

Five field stars, whose spectra showed no evidence of rotation, were also observed; their spectra were used in order to find the radial velocities of the Globular Cluster stars and as templates for the cross-correlations. These stars were selected on the basis of their atmospheric parameters in order to be as similar as possible to the program stars (see Gratton et al. 2001; Carretta et al. 2003, in preparation): G 4-37, G 126-62, G 141-019 for NGC 6397 and NGC 6752; HD 121004 and HD 120559 for NGC 104. The atmospheric parameters for the templates are given in Table 1 . We also observed several field stars with already studied rotational velocities in a range $\sim 9-80 \mathrm{~km} \mathrm{~s}^{-1}$. More details on these stars can be found in Sect. 4.

The spectra have a resolution of $R=\frac{\lambda}{F W H M} \simeq 50000$; data were obtained using a dichroic beamsplitter that provides useful spectra for both the blue and red arms of UVES. The signal-to-noise ratios span from 20 to over 100, with most of them around $\sim 40$. Except for stars \#201600 and \#435 of NGC 104, which were observed twice at 2 months distance, we did not collect multi-epoch observations. For further details on the observations, see Gratton et al. (2001). Extractions of the spectra for the observed stars were performed using the UVES pipeline.

In addition to the stars we observed, we also used as a calibrator in our analysis the UVES blue spectrum of the slowly rotating star CD -3018140 ( $\left.v_{\text {rot }} \sin i \sim 2 \mathrm{~km} \mathrm{~s}^{-1}\right)$ kindly provided by $\mathrm{P}$. Nissen.

\section{Data analysis}

\subsection{Cross-correlations}

We used only the blue part of the spectra, as it is not affected by telluric lines and have stronger signatures in the

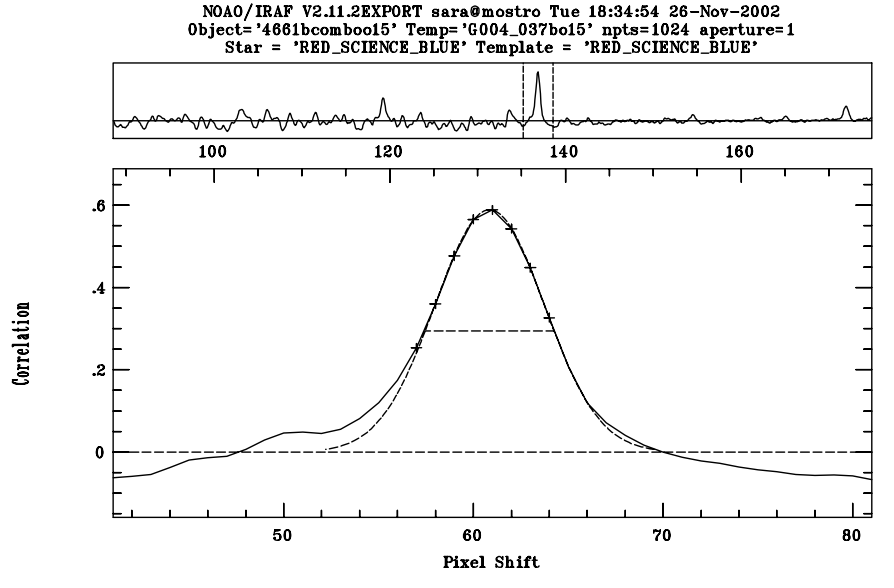

Fig. 1. Cross-correlation function of the 115th order of the spectrum of NGC 6752 star \#4661 versus G 4-37. The upper horizontal axis gives the scale in $\mathrm{km} \mathrm{s}^{-1}$ the lower one in pixels.

cross-correlations. The spectra of the object stars (calibrators and cluster stars) and those of templates have then been divided into 32 single orders. We discarded 5 of them for the metal poor stars and 11 in the NGC 104-47 Tuc ones, as within their range fell strong features (e.g. $\mathrm{H}$ lines or $\mathrm{Ca}$ II $\mathrm{H}$ and $\mathrm{K}$ lines), and these would have made them unsuitable to be used for rotational velocity determination. Each one of the remaining orders was first continuum fitted with a spline function of order 3 , and then cross-correlated against the corresponding orders of the template spectra from the appropriate group. We used the cross-correlation program $f x c o r$ from the IRAF ${ }^{1}$ noao.rv package. The cross-correlation peak was fitted using a Gaussian curve; this approximation is very reasonable as long as the rotational velocity is quite low.

An example of the fitting of a cross-correlation peak is given in Fig. 1, where we show the cross-correlation of the 115th order (4044-4068 $\AA$ ) of the spectrum of star \#4661 in NGC 6752 versus template G 4-37. As it can be seen in the figure, the Gaussian fit is a very good approximation in this case.

For each spectrum a total of 27 cross-correlations for the metal poor stars and 21 for the NGC 104-47 Tuc stars were obtained; for each one of these we measured the values of the $F W H M$, the relative velocity, and $R$ which is a goodness of the fit parameter (see Tonry \& Davis 1979).

\subsection{FWHM computation}

We computed the means and $\sigma$ 's for the $F W H M$ s and relative velocities iteratively, weighing on the basis of the $R$ parameter and discarding those cross-correlations for which the relative velocity was off from its mean value by more than $\sim 50 \mathrm{~km} \mathrm{~s}^{-1}$. Table 2 lists the resulting mean values for the FWHMs and their $\sigma$ 's. Both the individual values for the means from the cross-correlations with each of the templates, and the mean $F W H M$ from all the templates are given. The mean value for the

${ }^{1}$ IRAF is distributed by the National Optical Astronomy Observatories, which are operated by the Association of Universities for Research in Astronomy, Inc., under cooperative agreement with the National Science Foundation. 
Table 2. FWHMs fit from the cross-correlations and radial velocity for program stars. Star ID's are from Grundahl et al. (2000).

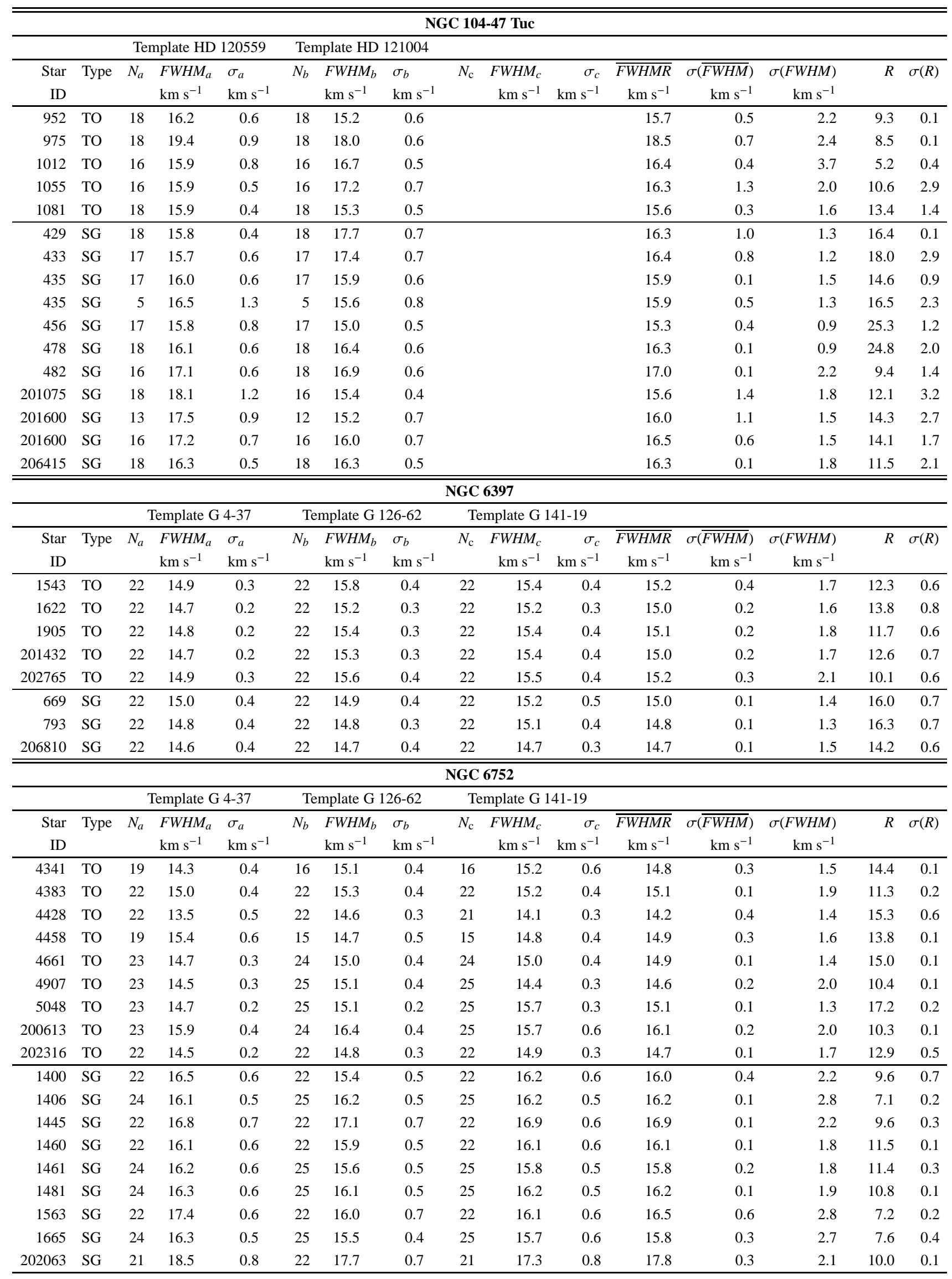


Table 3. FWHM's for templates (see text for details).

\begin{tabular}{|c|c|c|c|c|c|c|c|c|c|c|c|c|c|}
\hline & \multicolumn{3}{|c|}{ Template G 4-37 } & \multicolumn{3}{|c|}{ Template G 126-62 } & \multicolumn{3}{|c|}{ Template G 141-19 } & \multirow[b]{2}{*}{$\begin{array}{c}\overline{F W H M R} \\
\mathrm{~km} \mathrm{~s}^{-1}\end{array}$} & \multirow[b]{2}{*}{$\begin{array}{l}\sigma_{\overline{F W H M}} \\
\mathrm{~km} \mathrm{~s}^{-1}\end{array}$} & \multirow[b]{2}{*}{$R$} & \multirow[b]{2}{*}{$\sigma_{R}$} \\
\hline $\begin{array}{l}\text { Star } \\
\text { ID }\end{array}$ & $N_{a}$ & $\begin{array}{c}F W H M_{a} \\
\mathrm{~km} \mathrm{~s}^{-1}\end{array}$ & $\begin{array}{c}\sigma_{a} \\
\mathrm{~km} \mathrm{~s}^{-1}\end{array}$ & $N_{b}$ & $\begin{array}{c}F W H M_{b} \\
\mathrm{~km} \mathrm{~s}^{-1}\end{array}$ & $\begin{array}{c}\sigma_{b} \\
\mathrm{~km} \mathrm{~s}^{-1}\end{array}$ & $N_{c}$ & $\begin{array}{c}F W H M_{c} \\
\mathrm{~km} \mathrm{~s}^{-1}\end{array}$ & $\begin{array}{c}\sigma_{c} \\
\mathrm{~km} \mathrm{~s}^{-1}\end{array}$ & & & & \\
\hline G 4-37 & & & & 26 & 14.9 & 0.4 & 25 & 14.3 & 0.3 & 14.6 & 0.3 & 159.8 & 1.1 \\
\hline G $126-62$ & 25 & 14.9 & 0.4 & & & & 25 & 14.9 & 0.4 & 14.9 & 0.3 & 180.2 & 1.4 \\
\hline \multirow[t]{2}{*}{ G 141-19 } & 25 & 14.3 & 0.3 & 25 & 14.9 & 0.4 & & & & 14.6 & 0.3 & 199.2 & 1.4 \\
\hline & Ten & plate HD & 120559 & Tem & plate HD & 21004 & & & & & & & \\
\hline $\begin{array}{c}\text { Star } \\
\text { ID }\end{array}$ & $N_{a}$ & $\begin{array}{c}F W H M_{a} \\
\mathrm{~km} \mathrm{~s}^{-1}\end{array}$ & $\begin{array}{c}\sigma_{a} \\
\mathrm{~km} \mathrm{~s}^{-1}\end{array}$ & $N_{b}$ & $\begin{array}{c}F W H M_{b} \\
\mathrm{~km} \mathrm{~s}^{-1}\end{array}$ & $\begin{array}{c}\sigma_{b} \\
\mathrm{~km} \mathrm{~s}^{-1}\end{array}$ & $N_{c}$ & $\begin{array}{c}F W H M_{c} \\
\mathrm{~km} \mathrm{~s}^{-1}\end{array}$ & $\begin{array}{c}\sigma_{c} \\
\mathrm{~km} \mathrm{~s}^{-1}\end{array}$ & $\begin{array}{c}\overline{F W H M R} \\
\mathrm{~km} \mathrm{~s}^{-1}\end{array}$ & $\begin{array}{l}\sigma_{\overline{F W H M}} \\
\mathrm{~km} \mathrm{~s}^{-1}\end{array}$ & $R$ & $\sigma_{R}$ \\
\hline HD 120559 & & & & 15 & 18.0 & 0.4 & & & & 15.9 & 0.4 & 19.2 & 2.9 \\
\hline HD 121004 & 15 & 18.0 & 0.4 & & & & & & & 15.9 & 0.4 & 19.2 & 2.9 \\
\hline
\end{tabular}

Table 4. Heliocentric radial velocities for template stars compared to those in the literature.

\begin{tabular}{|c|c|c|c|c|c|}
\hline $\begin{array}{l}\text { Star } \\
\text { ID }\end{array}$ & $\begin{array}{r}V_{\mathrm{rad}} \\
\mathrm{km} \mathrm{s}^{-1}\end{array}$ & $\begin{array}{c}\sigma \\
\mathrm{km} \mathrm{s}^{-1}\end{array}$ & $\begin{array}{r}V_{\mathrm{rad}} \\
\mathrm{km} \mathrm{s}^{-1}\end{array}$ & $\begin{array}{c}\sigma \\
\mathrm{km} \mathrm{s}^{-1}\end{array}$ & MJD \\
\hline G 4-37 & -108.1 & 0.2 & $-108.5^{a}$ & 1.0 & 52151.425 \\
\hline G 126-62 & -297.0 & 0.2 & $-296.8^{b}$ & 1.0 & 52152.165 \\
\hline G $141-19$ & 84.3 & 0.1 & $84.3^{b}$ & 1.0 & 52152.009 \\
\hline HD 120559 & 12.8 & 0.1 & $13.4^{c}$ & & 51710.955 \\
\hline HD 121004 & 245.5 & 0.2 & $243.8^{c}$ & & 51712.960 \\
\hline
\end{tabular}

${ }^{a}$ Carney et al. (1994); ${ }^{b}$ Latham et al. (1988); ${ }^{c}$ Evans (1967).

$R$ parameter, calculated over the orders left after the aforementioned radial velocity selection, is also given. The value listed as $\sigma(F W H M)$ is the error on the fit of a single cross-correlation peak and has been calculated using the $\sigma(F W H M)-R$ relation appropriately calibrated on a set of calibration stars, as described in Sect. 3.4.

Then, using a very similar procedure, we calculated the contribution due to the templates. In fact, in order to estimate accurately the Doppler broadening of the program stars, it is important to model the contribution due to the template to the cross-correlation FWHMs. The subtraction of the template contribution from the FWHMs allows to take into account all the sources of Doppler broadening in the template spectrum, therefore, even though the spectra of the template stars do not show any evidence of considerable rotation, if the template were rotating with a small value of $v_{\text {rot }} \sin i$, its use in the cross-correlations would not affect our results. We performed cross-correlations of each of the orders for each one of the templates against the corresponding orders from the other templates of the same group (i.e. we did not take into account the auto-correlations because they would systematically underestimate the width of the peaks as the noise would be perfectly correlated). We calculated the mean values for the $F W H M$ s and $R$ parameter as described for the program stars. The results are listed in Table 3.

\subsection{Radial velocity determinations}

We obtained the mean apparent radial velocities using the same discarding and weighing scheme described in Sect. 3.2. We calculated the heliocentric velocity by adding up the appropriate template apparent velocity, as calculated from the measurement of the shifts of several spectral lines, and the barycenter correction. The values of radial velocity so obtained ( 3 for each of the stars of NGC 6397 and NGC 6752 and two for those of 47 Tuc) were then averaged weighing them on the basis of the respective error, which is the quadratic sum of the $\sigma$ obtained from the weighed order to order scatter of the apparent radial velocity, and the error of the radial velocity of the template (see Table 4). Table 5 lists the heliocentric radial velocities for the program stars, as obtained from the different templates and also the mean value. The radial velocities for the templates are shown in Table 4, together with the values published in the literature.

The resulting mean velocities for the clusters are listed in Table 6. Star \#1055 has been excluded from the computation of the mean velocity for NGC 104 because, as it evident from the measured radial velocity, it is obviously a non-member. Moreover, the radial velocities for the two stars observed during different observing runs, \#435 and \#201600 were assumed to be the average of the two determinations, so at the end the average velocity for NGC 104 is calculated over 13 stars.

\subsection{From FWHM's to $v_{\text {rot }} \sin i$}

In order to estimate the rotational velocities for the program stars we must take into account that the FWHM of the crosscorrelation peak gives a measure of the quadratic sum of the contributions coming from different sources:

$F W H M=\sqrt{t^{2}+r^{2}+m^{2}+M^{2}+T^{2}+i^{2}}$,

where $t$ is the contribution given to the calculated $F W H M$ by the template (which include micro and macro-turbulence, thermal broadening and instrumental profile for the template) and $r, m, M, T$ and $i$ are, respectively, the Doppler broadenings due to: the rotation of the object, the micro-turbulent velocity, the 
Table 5. Heliocentric radial velocities from the cross-correlations for program stars.

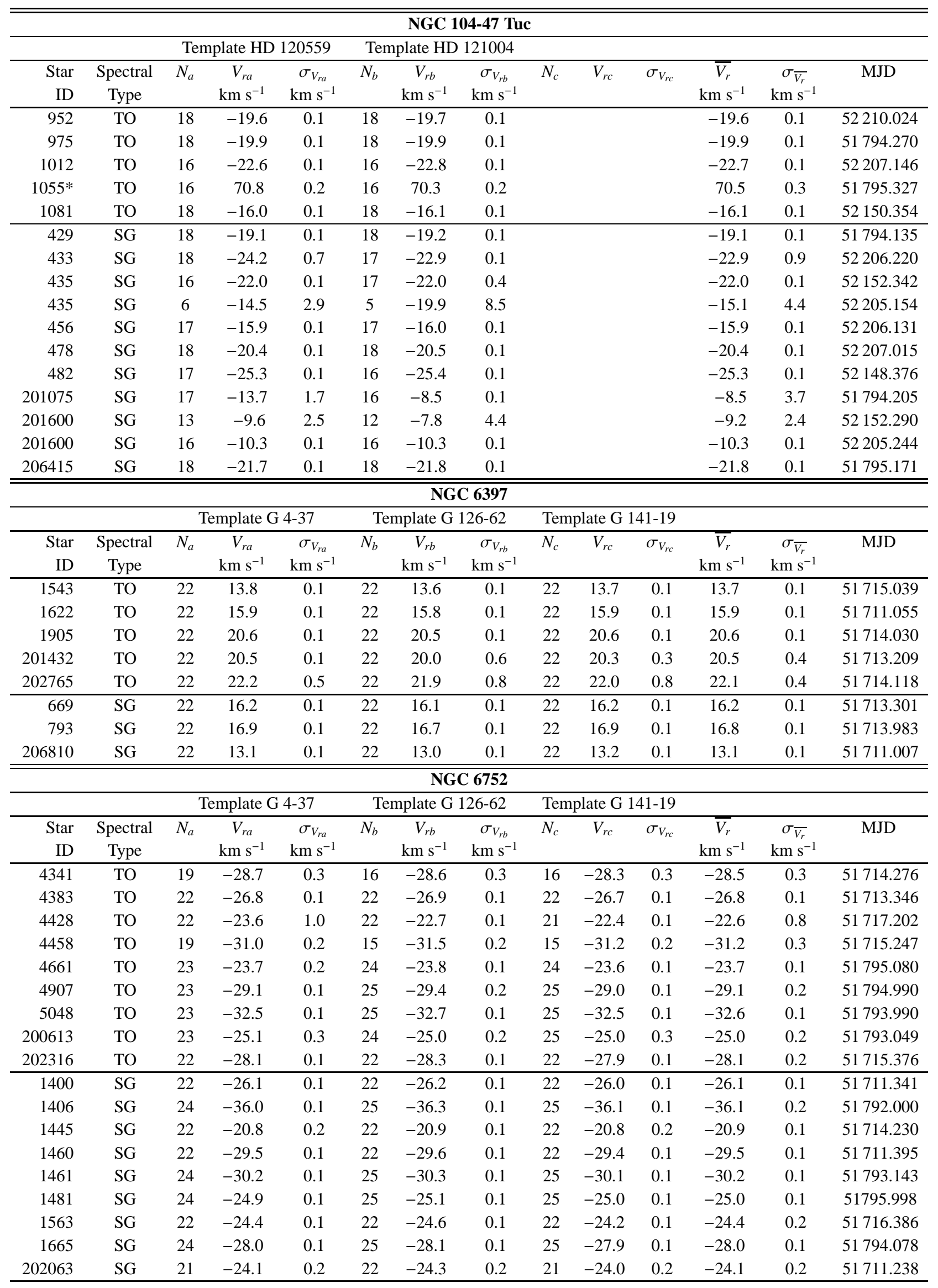


Table 6. Mean heliocentric radial velocities for the clusters. The values in Cols. 2, 3 and 4, are, respectively, the number of stars of our sample, the mean radial velocity and its error. Columns 5 and 6 show the results published in the literature (Harris 1996).

\begin{tabular}{lccccc}
\hline \hline Cluster & $\begin{array}{c}N \\
\text { ID }\end{array}$ & $\begin{array}{c}V_{\text {rad }} \\
\mathrm{km} \mathrm{s}^{-1}\end{array}$ & $\begin{array}{c}\sigma\left(\mathrm{V}_{\text {rad }}\right) \\
\mathrm{km} \mathrm{s}^{-1}\end{array}$ & $\begin{array}{c}\mathrm{V}_{\text {rad,lit }} \\
\mathrm{km} \mathrm{s}^{-1}\end{array}$ & $\begin{array}{c}\sigma\left(\mathrm{V}_{\text {rad }}\right)_{\text {lit }} \\
\mathrm{km} \mathrm{s}^{-1}\end{array}$ \\
\hline NGC 104 & 13 & -18.8 & 1.1 & -18.7 & 0.2 \\
NGC 6397 & 8 & 16.6 & 1.0 & 18.9 & 0.1 \\
NGC 6752 & 18 & -27.1 & 0.9 & -27.9 & 0.8 \\
\hline
\end{tabular}

macro-turbulent velocity, the thermal and instrumental broadenings. Therefore, from Eq. (1) we get:

$r=\sqrt{F W H M^{2}-\left[t^{2}+m^{2}+M^{2}+T^{2}+i^{2}\right]}$,

and, assuming that $\alpha$ is the coefficient which links the rotational velocity, $v_{\text {rot }} \sin i$ to $r$, the contribution to the cross-correlation peak due to rotation, we get:

$\left.v_{\mathrm{rot}} \sin i=\alpha \sqrt{F W H M^{2}-\left[t^{2}+m^{2}+M^{2}+T^{2}+i^{2}\right.}\right]$.

This coefficient must be calibrated and in order to do this we used a set of nine stars with already studied rotational velocities. These stars, with the exception of the slowest rotating one, $\mathrm{CD}-3018140$, which is however a metal poor star $([\mathrm{Fe} / \mathrm{H}]=-1.9 \mathrm{dex}$, Nissen et al. 2002), are quite hot, thus few lines are present in their spectra which thus mimic those of metal poor stars. Therefore, we used the metal poor templates in the cross correlations. We calculated the resulting FWHM, $R$ and $\sigma$ 's as described in Sect. 3.2. The results of such process are given in Table 7 .

Since these stars do not have published high resolution abundance analysis (again, with the exception of CD -30 18140, whose atmospheric parameters are $T_{\text {eff }}=$ $6274 \mathrm{~K}, \log g=4.1 \mathrm{dex},[\mathrm{Fe} / \mathrm{H}]=-1.9 \mathrm{dex} v_{\text {micro }}=1.5 \mathrm{~km} \mathrm{~s}^{-1}$ according to Nissen et al. 2002), we adopted for them the temperatures obtained from published photometry and Alonso et al. (1999) relations, on the basis of the luminosity class $\log g$. On the basis of $T_{\text {eff }}$ and $\log g$ we adopted a reasonable value of $v_{\text {micro }}$ and calculated $v_{\text {therm }}$. The accuracy of these values is not important here, as their combined contribution is at most of $\sim 4 \mathrm{~km} \mathrm{~s}^{-1}$, while the slowest rotating star for which we do not have accurate measurements of the atmospheric parameters has a value of $v_{\text {rot }} \sin i \sim 9 \mathrm{~km} \mathrm{~s}^{-1}$. Quadratic subtraction of the terms due to thermal and micro-turbulent velocities affects the result only marginally for low values of $v_{\text {rot }} \sin i$, and is absolutely negligible for $v_{\text {rot }} \sin i>20 \mathrm{~km} \mathrm{~s}^{-1}$. The contribution due to the template has been estimated assuming that the template stars within each group are very similar in atmospheric parameters, therefore the measured $F W H M$ of the cross-correlation peak is just:

$$
\overline{F W H M}_{\text {template }}=\sqrt{2} t
$$

where $\overline{F W H M}_{\text {template }}$ is calculated as described in the previous subsection and the values are listed in Table 3.
We adopted the macro-turbulent velocity values following Gray (1984, 1992); although these values are not determined with high accuracy for three of the calibration stars of the sample which are of early F spectral type (HD 43045, HD 49434 and HD 55892) this is not crucial for the results obtained. In fact, as the value of $v_{\text {rot }} \sin i$ increases, the contribution of rotation to the measured value of the FWHM becomes predominant; thus even assuming very pessimistically an error of 50\% in the value of the macro-turbulent velocity adopted for these stars, this would affect the derived value of $r$ only by less than $1 \%$. The atmospheric parameters adopted for the calibration stars are given in Table 8.

We calculated the values for $r$ according to Eq. (2), from the resulting mean value of the FWHM's and using the atmospheric parameters showed in Table 8 . The resulting values of $r$ obtained are listed in Table 9. We also measured the rotational velocity for these stars (with the exception of CD -30 18140) using the Fourier transform method (Gray 1992). To do this we used the red part of the spectrum which is characterized by few and well isolated lines. We measured the rotational velocities from the following lines: Ca I at $6439.1 \AA$, $6449.8 \AA$ and Fe I $6065.8 \AA, 6678.0 \AA$, which are well isolated. We could not apply this method to $C D-3018140$ as the red spectrum was not available. The values given in Col. 6 of Table 9 are the mean of the four values obtained, while the errors listed in Col. 7 are the $\sigma$ around these mean values. We performed a fit of the $r$ value to the rotational velocities, both from the literature and those measured using the Fourier transform method. The errors take into account, beside the intrinsic scatter of the measurement of $F W H M$ for the stars, the uncertainties on the $F W H M$ of the templates $\left(0.3 \mathrm{~km} \mathrm{~s}^{-1}\right)$, $v_{\text {therm }}\left(0.1 \mathrm{~km} \mathrm{~s}^{-1}\right), v_{\text {micro }}\left(0.3 \mathrm{~km} \mathrm{~s}^{-1}\right), \zeta_{\mathrm{RT} \text {,macro }}\left(0.3 \mathrm{~km} \mathrm{~s}^{-1}\right)$ and on the instrumental profile $\left(0.1 \mathrm{~km} \mathrm{~s}^{-1}\right)$. Figure 2 gives a graphical representation of the results. The values obtained for the $\alpha$ coefficient is, in both cases, $\alpha=0.6$, while the rms's of the fits are $2.7 \mathrm{~km} \mathrm{~s}^{-1}$ for the fit with the literature results, and $2.0 \mathrm{~km} \mathrm{~s}^{-1}$ for the fit with the values measured with the Fourier Transform method.

As can be noticed from the graph, the fit is excellent up to $\sim 20 \mathrm{~km} \mathrm{~s}^{-1}$. Beyond that value there is a close correlation between the measured values of $r$ and the values of $v_{\text {rot }} \sin i$ published in the literature and with the values measured by us using the Fourier transform method. However, as can be noticed especially from the comparison with the values obtained using the Fourier transform value, which are determined with a higher degree of accuracy with respect to the literature values, our results suggest that for values of $v_{\text {rot }} \sin i$ larger than $\sim 50 \mathrm{~km} \mathrm{~s}^{-1}$, the cross-correlation method tends to overestimate the rotational velocities. The discrepancy at larger rotational velocities is intrinsic to the cross-correlation method. In fact, a Gaussian is a good approximation for the crosscorrelation function as long as the rotation is slow, and gets progressively worse as $v_{\text {rot }} \sin i$ increases. Nevertheless, this test clearly shows that our method supplies reliable estimates of the values of $v_{\text {rot }} \sin i$ in the range $\sim 2-50 \mathrm{~km} \mathrm{~s}^{-1}$.

Table 10 lists the heliocentric radial velocities resulting from our analysis for this sample of stars (Cols. 2 and 3), and compares them with the values published in the literature 
Table 7. FWHM's values from the cross-correlation fit for calibration stars.

\begin{tabular}{lccccccccccccc}
\hline \hline $\begin{array}{l}\text { Star } \\
\text { ID }\end{array}$ & $N_{a}$ & $\begin{array}{c}F W H M_{a} \\
\mathrm{~km} \mathrm{~s}^{-1}\end{array}$ & $\begin{array}{c}\sigma_{a} \\
\mathrm{~km} \mathrm{~s}^{-1}\end{array}$ & $\begin{array}{c}N_{b} \\
\mathrm{~km} \mathrm{~s}^{-1}\end{array}$ & $\begin{array}{c}\sigma_{b} \\
\mathrm{~km} \mathrm{~s}^{-1}\end{array}$ & $\begin{array}{c}N_{c} \\
\mathrm{kmWH}_{c} \\
\mathrm{~km} \mathrm{~s}^{-1}\end{array}$ & $\begin{array}{c}\sigma_{c} \\
\mathrm{~km} \mathrm{~s}^{-1}\end{array}$ & $\begin{array}{c}\overline{F W H M} \\
\mathrm{~km} \mathrm{~s}^{-1}\end{array}$ & $\begin{array}{c}\sigma(\overline{F W H M}) \\
\mathrm{km} \mathrm{s}^{-1}\end{array}$ & $R$ & $\sigma(R)$ \\
\hline HD 31623 & 12 & 104.7 & 7.1 & 25 & 112.2 & 3.6 & 25 & 113.5 & 4.2 & 111.7 & 2.1 & 6.22 & 0.91 \\
HD 34045 & 14 & 111.8 & 7.1 & 23 & 114.8 & 3.1 & 25 & 112.7 & 4.2 & 113.8 & 0.9 & 5.09 & 1.25 \\
HD 38393 & 18 & 22.6 & 1.3 & 26 & 20.9 & 0.5 & 26 & 21.8 & 0.6 & 21.4 & 0.4 & 3.99 & 1.97 \\
HD 42278 & 16 & 34.1 & 1.9 & 25 & 35.4 & 0.5 & 25 & 35.5 & 0.7 & 35.3 & 0.2 & 9.53 & 1.54 \\
HD 49434 & 10 & 158.5 & 11.2 & 19 & 128.6 & 3.2 & 21 & 137.4 & 4.4 & 133.1 & 5.8 & 7.65 & 0.53 \\
HD 55892 & 15 & 85.7 & 7.5 & 25 & 82.7 & 3.0 & 27 & 77.0 & 3.4 & 80.6 & 2.4 & 6.61 & 1.07 \\
HD 65228 & 18 & 28.2 & 1.3 & 26 & 28.1 & 0.7 & 26 & 27.8 & 0.5 & 27.9 & 0.1 & 7.71 & 1.39 \\
HD 71243 & 19 & 50.2 & 2.2 & 25 & 54.8 & 1.7 & 26 & 52.9 & 1.3 & 53.0 & 1.1 & 4.36 & 1.60 \\
CD -30 18140 & 15 & 12.9 & 0.2 & 15 & 13.4 & 0.2 & 15 & 13.3 & 0.3 & 13.2 & 0.2 & 18.31 & 0.29 \\
\hline
\end{tabular}

${ }^{a}$ To calculate $v_{\text {therm }}$ we used for $T_{\text {ecc }}=0.86 T_{\text {eff }}$.

(Cols. 4 and 5). We note that the spectrum of CD -30 18140 we used (courtesy of Poul Nissen) was already brought to its rest frame and could therefore not be used for this purpose. The results agree quite well within the errors, with the exception of HD 34045, which, however was labeled as a suspect double by Grenier et al. (1999). The good agreement is a further confirmation of the reliability of our radial velocities.

In order to obtain an estimate of the error on the single $F W H M$ fit, we calibrated the $R$ parameter- $\sigma(F W H M$ ) (where the $\sigma(F W H M)$ is the mean value of the order-to-order rms's resulting from the different templates) relation, which should follow the form $\sigma(F W H M) \propto \frac{1}{1+R}$ (Tonry \& Davis 1979). Since, as discussed above, the Gaussian fitting of the cross-correlation peak gives reasonable results up to $\sim 50 \mathrm{~km} \mathrm{~s}^{-1}$, we discarded from this fit the calibrating stars with $v_{\text {rot }} \sin i$ above this limit. As Fig. 3 shows, we find a quite close relationship and the best fit is obtained with the equation:

$\sigma(F W H M)=(23 \pm 3) \frac{1}{1+R} \mathrm{~km} \mathrm{~s}^{-1}$

with an rms of the fit rms $=0.2 \mathrm{~km} \mathrm{~s}^{-1}$. The latter equation is plotted in Fig. 3.

This equation is a calibration of the $R$ parameter- $\sigma(F W H M)$ relation; thus the $R$ parameter can be used to estimate errors on the single FWHM measurements. In fact the goodness of the fit parameter $R$ is returned for each of the cross-correlations by fxcor, thus in principle its value can be entered in Eq. (5) producing the corresponding value for the error on the fit of a single FWHM. The values listed in Col. 14 of Table 2 are obtained using this procedure. However, it should be kept in mind that the $R$ parameter- $\sigma(F W H M)$ relation, besides depending on the signal-to-noise ratio, is dependent on the spectral match between the object and the template star.

\subsection{Program stars}

The procedure outlined above for calculating $v_{\text {rot }} \sin i$ can be applied to the cluster stars; the values of micro-turbulent and thermal broadening were estimated on the basis of the atmospheric parameters found during the abundance analysis.
Table 8. Adopted atmospheric parameters for calibration stars.

\begin{tabular}{rcccc}
\hline \hline ID & SpType & $v_{\text {micro }}$ & $v_{\text {therm }}^{a}$ & $\zeta_{\text {RT,macro }}$ \\
\hline HD 31623 & F2 V & 1.6 & 1.3 & 7.2 \\
HD 34045 & F3 III & 1.8 & 1.3 & 11.0 \\
HD 38393 & F7 V & 1.4 & 1.3 & 5.3 \\
HD 42278 & F4 IV & 1.5 & 1.4 & 8.0 \\
HD 49434 & F1 V & 1.7 & 1.3 & 8.0 \\
HD 55892 & F0 IV & 1.7 & 1.3 & 9.0 \\
HD 65228 & F7/F8 II & 1.0 & 1.2 & 9.0 \\
HD 71243 & F5 V & 1.3 & 1.3 & 6.5 \\
CD -30 18140 & F7 V & 1.5 & 1.3 & 5.0 \\
\hline
\end{tabular}

${ }^{a}$ To calculate $v_{\text {therm }}$ we used for $T_{\text {ecc }}=0.86 T_{\text {eff }}$.

For NGC 6397 and NGC 6752 we used the values given in Gratton et al. (2001), for NGC 104-47 Tuc those from Carretta et al. (2003, in preparation). The corresponding values for the micro-turbulent and thermal velocity are given in Table 11. Macro-turbulent velocity estimates for this type of stars are not easily available in the literature. The values adopted for the calibrating stars from Gray $(1984,1992)$ are suited to Population I stars. Given the low metal content of the cluster stars, the atmosphere is more transparent and we can see deeper into in, thus the value of the macro-turbulent velocity is expected to be larger than the corresponding one for a Population I star of the same effective temperature and gravity.

Therefore, the values from Gray $(1984,1992)$ can be considered as lower limits to the true values of the macroturbulent velocities and, therefore, the values of $v_{\text {rot }} \sin i$ obtained adopting them are upper limits to the true $v_{\text {rot }} \sin i$. From statistical consideration, it is possible to further constrain the macro-turbulent velocity, as is discussed in Sect. 4.2. The adopted values for the clusters stars, which are, as discussed, lower limits, are listed in Table 11. 
Table 9. Values of $r$ found with our method for the field stars with previously studied rotation velocity.

\begin{tabular}{lrrrrrr}
\hline \hline \multicolumn{7}{c}{ Rotational velocities } \\
\hline Star & $\begin{array}{r}r \\
\mathrm{~km} \mathrm{~s}^{-1}\end{array}$ & $\begin{array}{r}\sigma(r) \\
\mathrm{km} \mathrm{s}^{-1}\end{array}$ & $\begin{array}{r}v_{\text {rot }} \sin i(\mathrm{~L}) \\
\mathrm{km} \mathrm{s}^{-1}\end{array}$ & $\begin{array}{r}\sigma(\mathrm{L}) \\
\mathrm{km} \mathrm{s}^{-1}\end{array}$ & $\begin{array}{r}v_{\text {rot }} \sin i(\mathrm{G}) \\
\mathrm{km} \mathrm{s}^{-1}\end{array}$ & $\begin{array}{r}\sigma(\mathrm{G}) \\
\mathrm{km} \mathrm{s}^{-1}\end{array}$ \\
\hline HD 31623 & 110.7 & 2.1 & $75.0^{a}$ & $10.0^{a}$ & 63.2 & 3.4 \\
HD 34045 & 112.5 & 0.9 & $75.0^{a}$ & $10.0^{a}$ & 70.1 & 3.0 \\
HD 38393 & 16.0 & 0.6 & $8.7^{b}$ & $0.8^{b}$ & 9.0 & 3.0 \\
HD 42278 & 31.8 & 0.3 & $18.0^{c}$ & $10.0^{c}$ & 16.7 & 3.1 \\
HD 49434 & 132.2 & 5.9 & $79.0^{d}$ & $10.0^{d}$ & 80.7 & 3.2 \\
HD 55892 & 79.0 & 2.4 & $50.0^{e}$ & $5.0^{e}$ & 47.0 & 3.6 \\
HD 65228 & 23.1 & 0.3 & $13.8^{f}$ & $1.0^{f}$ & 14.9 & 3.0 \\
HD 71243 & 50.6 & 1.2 & $28.6^{f}$ & $2.9^{f}$ & 26.2 & 3.0 \\
CD -30 18140 & 2.4 & 1.6 & $1.84^{g}$ & $0.2^{g}$ & & \\
\hline
\end{tabular}

${ }^{a}$ Uesugi \& Fakuda (1982); ${ }^{b}$ Soderblom et al. (1989); ${ }^{c}$ Abt \& Morrell (1995); ${ }^{d}$ Wolff \& Simon (1997); ${ }^{e}$ Balachandran (1990); ${ }^{f}$ de Medeiros \& Mayor (1999); ${ }^{g}$ Asplund et al. (2003), in preparation.

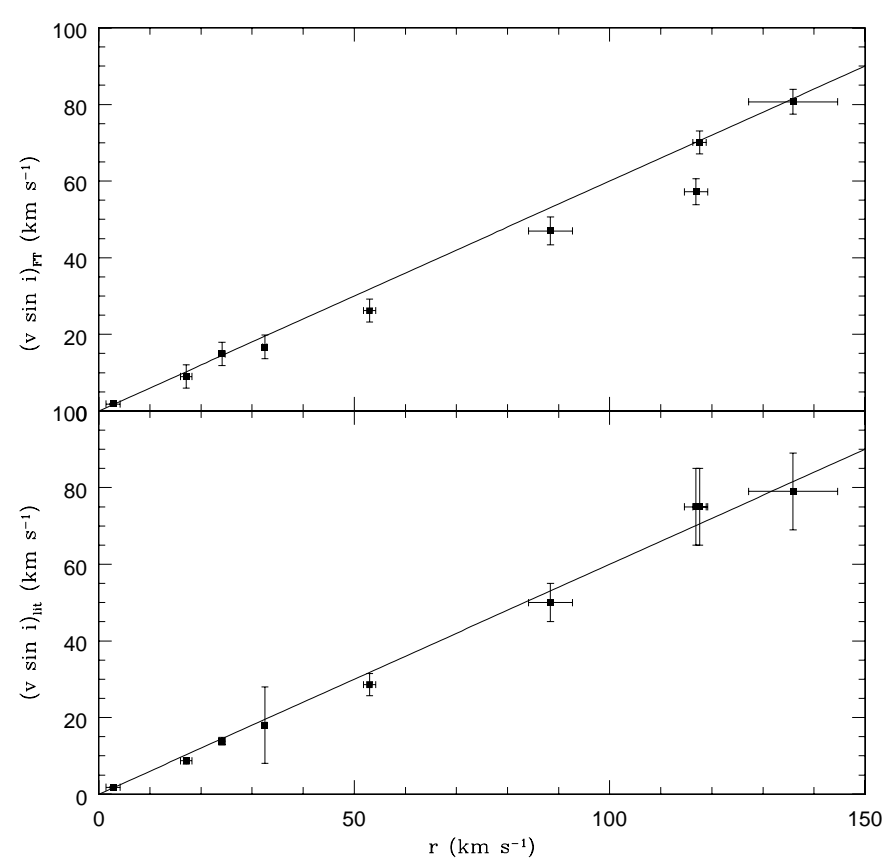

Fig. 2. $r$ values obtained as described in the text versus literature $v_{\text {rot }} \sin i$ (lower panel) and $v_{\text {rot }} \sin i$ measured by us using the Fourier Transform method (upper panel). The lines plotted are the best fit lines, and both have the same slope, within the errors, of $\alpha=0.6$.

\section{Results}

\subsection{Radial velocities}

\subsubsection{Template stars}

Table 4 lists the heliocentric radial velocities we calculated for the template stars together with those published in the literature (see Table 4 for references). Since G 4-37 and G 126-62 are known and studied spectroscopic binaries, the values used for comparison in Table 4 were calculated from the published orbital parameters (Latham et al. 1988). Our results are very consistent with those previously published; the mean offset is $0.5 \pm 0.7 \mathrm{~km} \mathrm{~s}^{-1}$.

\subsubsection{Cluster stars}

The resulting radial velocities for NGC 104 and NGC 6752, listed in Table 6, are in excellent agreement with data published in the literature (Harris 1996), while those for NGC 6397 seem to be smaller by about $2.3 \mathrm{~km} \mathrm{~s}^{-1}$, which is a little over $2 \sigma$. We notice that the procedure used to obtain the radial velocities was identical for all the program stars and that it leads to a nice agreement of the results for the other two clusters with the literature; we did not find any systematic offset in the radial velocity of the template stars with previous determinations. Thus, it seems unlikely that this discrepancy is due to systematic errors. On the other hand, NGC 6397 is the cluster for which we had the smallest sample of observed stars, so the difference might simply due to our sampling (the internal velocity dispersion in this cluster is of $\sim 3 \mathrm{~km} \mathrm{~s}^{-1}$, see Harris 1996).

\subsection{Rotational velocities}

The resulting values of $v_{\text {rot }} \sin i$ calculated as outlined in Sect. 3.5 are listed in Table 12. As in the case of the calibration stars, the errors take into account, beside the intrinsic scatter of the measurement of FWHM for the program stars, the uncertainties on the $F W H M$ of the templates $\left(0.3\right.$ and $0.4 \mathrm{~km} \mathrm{~s}^{-1}$ respectively for the metal poor and metal rich templates), $v_{\text {therm }}\left(0.1 \mathrm{~km} \mathrm{~s}^{-1}\right), v_{\text {micro }}\left(0.3 \mathrm{~km} \mathrm{~s}^{-1}\right), \zeta_{\mathrm{RT} \text {,macro }}\left(0.3 \mathrm{~km} \mathrm{~s}^{-1}\right)$ and on the instrumental profile $\left(0.1 \mathrm{~km} \mathrm{~s}^{-1}\right)$. A graphical representation of our results is given in Fig. 4, which shows the computed values for $v_{\text {rot }} \sin i$ versus $M_{V}$, using the distance moduli from Carretta et al. (2000). As it can be noticed from this figure and also from Table 12, the distribution of the 
Table 10. Heliocentric radial velocities found with our method for the field stars with well studied rotation velocity compared to those published in the literature.

\begin{tabular}{|c|c|c|c|c|c|c|c|c|c|c|}
\hline & \multicolumn{2}{|c|}{ Template G 4-37 } & \multicolumn{2}{|c|}{ Template G 126-62 } & \multicolumn{2}{|c|}{ Template G 141-19 } & \multirow[b]{2}{*}{$\begin{array}{c}\overline{V_{r}} \\
\mathrm{~km} \mathrm{~s}^{-1}\end{array}$} & \multirow[b]{2}{*}{$\begin{array}{c}\sigma_{\overline{V_{r}}} \\
\mathrm{~km} \mathrm{~s}^{-1}\end{array}$} & \multicolumn{2}{|c|}{ Literature values } \\
\hline $\begin{array}{r}\text { Star } \\
\text { ID } \\
\end{array}$ & $\begin{array}{c}V_{r a} \\
\mathrm{~km} \mathrm{~s}^{-1}\end{array}$ & $\begin{array}{c}\sigma_{V_{r a}} \\
\mathrm{~km} \mathrm{~s}^{-1}\end{array}$ & $\begin{array}{c}V_{r b} \\
\mathrm{~km} \mathrm{~s}^{-1} \\
\end{array}$ & $\begin{array}{c}\sigma_{V_{r b}} \\
\mathrm{~km} \mathrm{~s}^{-1}\end{array}$ & $\begin{array}{c}V_{r c} \\
\mathrm{~km} \mathrm{~s}^{-1} \\
\end{array}$ & $\begin{array}{c}\sigma_{V_{r c}} \\
\mathrm{~km} \mathrm{~s}^{-1}\end{array}$ & & & $\begin{array}{c}V_{r, \text { lit }} \\
\mathrm{km} \mathrm{s}^{-1}\end{array}$ & $\begin{array}{c}\sigma_{V_{r, \text { lit }}} \\
\mathrm{km} \mathrm{s}^{-1} \\
\end{array}$ \\
\hline HD 31623 & 4.8 & 1.8 & 10.9 & 1.2 & 13.5 & 1.6 & 10.3 & 0.8 & $13.3^{a}$ & \\
\hline HD 34045 & 20.3 & 2.0 & 29.3 & 1.3 & 32.7 & 1.7 & 28.4 & 0.9 & $28.62^{b, e}$ & 0.70 \\
\hline HD 38393 & -9.5 & 0.2 & -9.5 & 0.2 & -9.4 & 0.2 & -9.5 & 0.1 & $-9.72^{c}$ & 0.1 \\
\hline HD 42278 & -2.7 & 0.3 & -2.6 & 0.2 & -2.2 & 0.2 & -2.5 & 0.1 & & \\
\hline HD 49434 & -21.2 & 2.3 & -18.9 & 1.5 & -10.9 & 1.9 & -16.9 & 1.0 & $-14.00^{b, e}$ & 0.89 \\
\hline HD 55892 & 0.6 & 1.5 & 1.8 & 0.6 & 4.0 & 0.8 & 2.4 & 0.5 & $-0.13^{b, f}$ & 0.57 \\
\hline HD 65228 & 12.6 & 0.3 & 12.4 & 0.2 & 12.8 & 0.2 & 12.6 & 0.1 & $12.96^{d}$ & 0.13 \\
\hline HD 71243 & -14.8 & 0.5 & -13.6 & 0.2 & -13.1 & 0.3 & -13.6 & 0.2 & $-13.28^{d}$ & 0.3 \\
\hline CD -30 18140 & $0.0^{g}$ & 0.1 & 0.0 & 0.1 & 0.0 & 0.1 & 0.0 & 0.1 & & \\
\hline
\end{tabular}

${ }^{a}$ Duflot et al. (1995); ${ }^{b}$ Grenier et al. (1999); ${ }^{c}$ Skuljan et al. (2000); ${ }^{d}$ de Medeiros \& Mayor (1999); ${ }^{e}$ Suspect double; ${ }^{f}$ Probable double; ${ }^{g}$ Spectrum was already zero velocity shifted.

Table 11. Adopted atmospheric parameters for program stars.

\begin{tabular}{|c|c|c|c|c|c|c|c|}
\hline \multicolumn{8}{|c|}{ NGC 104-47 Tuc } \\
\hline & $\begin{array}{c}\text { Spectral } \\
\text { type }\end{array}$ & $\begin{array}{c}T_{\text {eff }} \\
\mathrm{K}\end{array}$ & $\begin{array}{c}\log g \\
\operatorname{dex}\end{array}$ & $\begin{array}{c}{[\mathrm{Fe} / \mathrm{H}]} \\
\operatorname{dex}\end{array}$ & $\begin{array}{c}v_{\text {micro }} \\
\mathrm{km} \mathrm{s}^{-1}\end{array}$ & $\begin{array}{c}v_{\text {therm }}^{a} \\
\mathrm{~km} \mathrm{~s}^{-1}\end{array}$ & $\begin{array}{l}\zeta_{\mathrm{RT} \text {,macro }} \\
\mathrm{km} \mathrm{s}^{-1}\end{array}$ \\
\hline & TO & 5832 & 4.05 & -0.62 & 1.07 & 1.3 & 4.0 \\
\hline 429 & SG & 5081 & 3.84 & -0.61 & 1.04 & 1.2 & 4.0 \\
\hline 433 & SG & 5106 & 3.84 & -0.74 & 1.05 & 1.2 & 4.0 \\
\hline 435 & SG & 5190 & 3.84 & -0.63 & 0.00 & 1.2 & 4.0 \\
\hline 456 & SG & 5142 & 3.84 & -0.68 & 0.50 & 1.2 & 4.0 \\
\hline 478 & SG & 5118 & 3.84 & -0.56 & 0.00 & 1.2 & 4.0 \\
\hline 482 & SG & 5150 & 3.84 & -0.64 & 0.70 & 1.2 & 4.0 \\
\hline 201075 & SG & 5165 & 3.84 & -0.64 & 0.70 & 1.2 & 4.0 \\
\hline 201600 & SG & 5160 & 3.84 & -0.61 & 0.70 & 1.2 & 4.0 \\
\hline 206415 & SG & 5112 & 3.84 & -0.67 & 1.05 & 1.2 & 4.0 \\
\hline \multicolumn{8}{|c|}{ NGC 6397} \\
\hline & Spectral & $T_{\text {eff }}$ & $\log g$ & {$[\mathrm{Fe} / \mathrm{H}]$} & $v_{\text {micro }}$ & $v_{\text {therm }}^{a}$ & $\zeta_{\mathrm{RT} \text {,macro }}$ \\
\hline & type & K & $\operatorname{dex}$ & dex & $\mathrm{km} \mathrm{s}^{-1}$ & $\mathrm{~km} \mathrm{~s}^{-1}$ & $\mathrm{~km} \mathrm{~s}^{-1}$ \\
\hline & TO & 6476 & 4.1 & -2.04 & 1.32 & 1.4 & 6.0 \\
\hline & SG & 5478 & 3.4 & -2.04 & 1.32 & 1.3 & 4.5 \\
\hline \multicolumn{8}{|c|}{ NGC 6752} \\
\hline & Spectral & $T_{\text {eff }}$ & $\log g$ & {$[\mathrm{Fe} / \mathrm{H}]$} & $v_{\text {micro }}$ & $v_{\text {therm }}^{a}$ & $\zeta_{\mathrm{RT} \text {,macro }}$ \\
\hline & type & K & $\operatorname{dex}$ & dex & $\mathrm{km} \mathrm{s}^{-1}$ & $\mathrm{~km} \mathrm{~s}^{-1}$ & $\mathrm{~km} \mathrm{~s}^{-1}$ \\
\hline & TO & 6226 & 4.28 & -1.43 & 0.70 & 1.4 & 5.3 \\
\hline & SG & 5347 & 3.54 & -1.43 & 1.47 & 1.3 & 4.5 \\
\hline
\end{tabular}

${ }^{a}$ To calculate $v_{\text {therm }}$ we used for $T_{\text {ecc }}=0.86 T_{\text {eff }}$.

values of $v_{\text {rot }} \sin i$ are flat within each cluster, and appear to be very similar. It is also noteworthy that the results for the two stars in NGC 104 which were observed during 2 different runs, \#435 and \#201600, are compatible within the observational errors. This result is a confirmation of the stability of the method and of the independence of the results from the signalto-noise ratio which was quite different for the two spectra. Averages of $v_{\text {rot }} \sin i$ over the TO and SG for each of the 


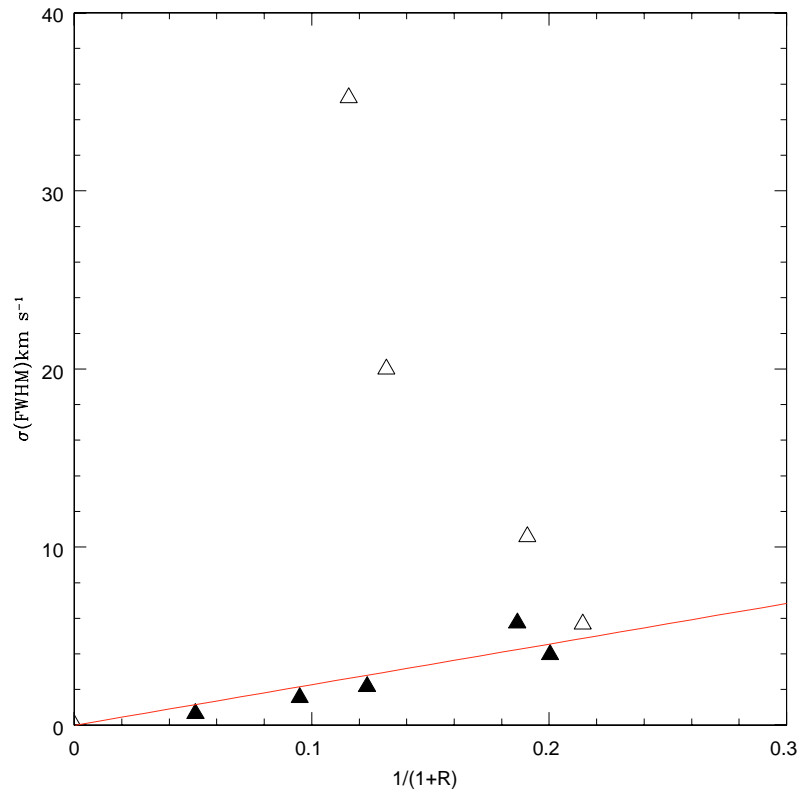

Fig. 3. Relationship between the $R$ parameter and the $\sigma(F W H M)$ of the measured $F W H M$ distribution. The plotted line represent the best fit equation after the exclusion of stars with $v_{\text {rot }} \sin i>50 \mathrm{~km} \mathrm{~s}^{-1}$ (open symbols) from the sample.
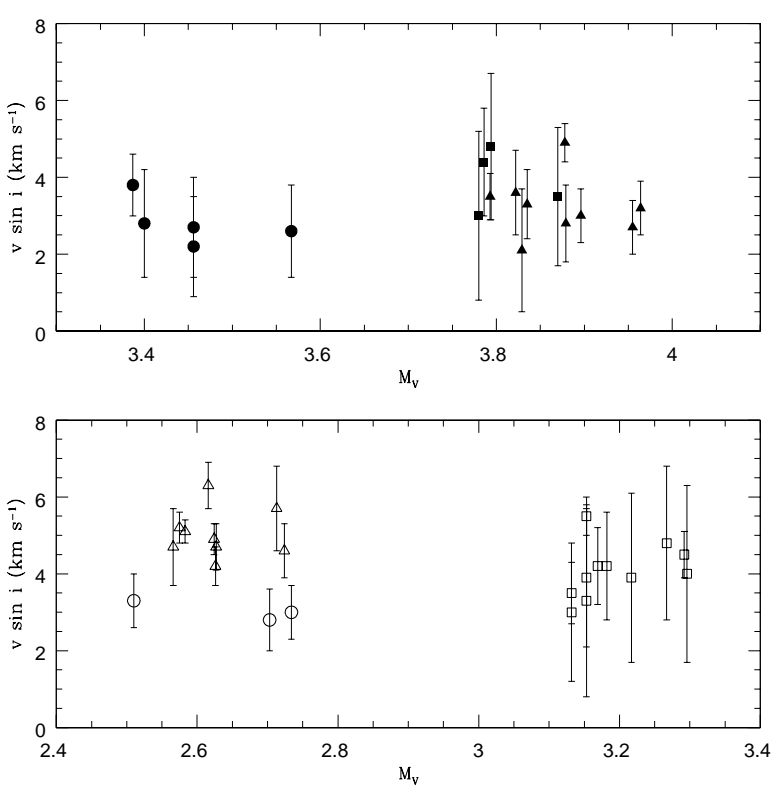

Fig. 4. Values for the rotational velocities of the program stars. Top panel: $v_{\text {rot }} \sin i$ for the TO stars, the squares represent the NGC 104 stars, the triangles those of NGC 6752 and the circles those of NGC 6397. Bottom panel: as top panel for SG stars.

3 clusters are given in Table 12. The mean values of $v_{\text {rot }} \sin i$ over the TO over all the stars are:

$$
\begin{array}{ll}
{\overline{v_{\text {rot }} \sin i_{\mathrm{TO}}}}=3.5 \pm 0.2 \mathrm{~km} \mathrm{~s}^{-1} & \mathrm{rms}=1.0 \mathrm{~km} \mathrm{~s}^{-1} \\
{\overline{v_{\text {rot }} \sin i_{\mathrm{SG}}}}_{\text {r }}=4.7 \pm 0.2 \mathrm{~km} \mathrm{~s}^{-1} & \mathrm{rms}=0.7 \mathrm{~km} \mathrm{~s}^{-1} .
\end{array}
$$

The flatness of the distribution and its small scatter suggest that the contribution given by rotation to the measured Doppler broadening is not large. If the contribution of the rotation were
Table 12. Values of the upper limits of $v_{\text {rot }} \sin i$ obtained after the subtraction of the template contribution and the broadening due to micro

\begin{tabular}{|c|c|c|c|c|c|}
\hline $\begin{array}{l}\text { Star } \\
\text { ID }\end{array}$ & $\begin{array}{l}v_{\text {rot }} \sin i \\
\mathrm{~km} \mathrm{~s}^{-1}\end{array}$ & $\begin{array}{l}\sigma \\
\mathrm{km} \mathrm{s}^{-1}\end{array}$ & $\begin{array}{l}\text { Star } \\
\text { ID }\end{array}$ & $\begin{array}{l}v_{\text {rot }} \sin i \\
\mathrm{~km} \mathrm{~s}^{-1}\end{array}$ & $\begin{array}{l}\sigma \\
\mathrm{km} \mathrm{s}^{-1}\end{array}$ \\
\hline \multicolumn{6}{|c|}{ NGC 104-47 Tuc } \\
\hline 952 & 4.4 & 1.4 & 429 & 3.3 & 2.5 \\
\hline 975 & 3.0 & 2.2 & 433 & 3.9 & 1.8 \\
\hline 1012 & 4.8 & 1.9 & 435 & 4.2 & 1.4 \\
\hline \multirow[t]{8}{*}{1081} & 3.5 & 1.8 & 435 & 5.5 & 0.5 \\
\hline & & & 456 & 3.9 & 2.2 \\
\hline & & & 478 & 4.0 & 2.3 \\
\hline & & & 482 & 4.5 & 0.6 \\
\hline & & & 201075 & 4.8 & 2.0 \\
\hline & & & 201600 & 3.0 & 1.8 \\
\hline & & & 201600 & 3.5 & 0.8 \\
\hline & & & 206415 & 4.2 & 1.0 \\
\hline \multicolumn{3}{|c|}{$\begin{array}{l}\overline{v_{\mathrm{rot}} \sin i_{\mathrm{TO}}}=4.0 \pm 0.4 \mathrm{~km} \mathrm{~s}^{-1} \\
\mathrm{rms}_{\mathrm{TO}}=0.8 \mathrm{~km} \mathrm{~s}^{-1}\end{array}$} & \multicolumn{3}{|c|}{$\begin{array}{l}\overline{v_{\mathrm{rot}} \sin i_{\mathrm{SG}}}=4.6 \pm 0.3 \mathrm{~km} \mathrm{~s}^{-1} \\
\mathrm{rms}_{\mathrm{SG}}=0.9 \mathrm{~km} \mathrm{~s}^{-1}\end{array}$} \\
\hline \multicolumn{6}{|c|}{ NGC 6397} \\
\hline 1543 & 2.8 & 1.4 & 669 & 3.3 & 0.7 \\
\hline 1622 & 3.8 & 0.8 & 793 & 3.0 & 0.7 \\
\hline 1905 & 2.6 & 1.2 & 206810 & 2.8 & 0.8 \\
\hline 201432 & 2.2 & 1.3 & & & \\
\hline 202765 & 2.7 & 1.3 & & & \\
\hline \multicolumn{3}{|c|}{$\begin{array}{l}\bar{v}_{\mathrm{rot}} \sin i_{\mathrm{TO}}=3.1 \pm 0.3 \mathrm{~km} \mathrm{~s}^{-1} \\
\mathrm{rms}_{\mathrm{TO}}=0.7 \mathrm{~km} \mathrm{~s}^{-1}\end{array}$} & \multicolumn{3}{|c|}{$\begin{array}{l}\bar{v}_{\mathrm{rot}} \sin i_{\mathrm{SG}}=3.1 \pm 0.1 \mathrm{~km} \mathrm{~s}^{-1} \\
\mathrm{rms}_{\mathrm{SG}}=0.2 \mathrm{~km} \mathrm{~s}^{-1}\end{array}$} \\
\hline \multicolumn{6}{|c|}{ NGC 6752} \\
\hline 4341 & 2.1 & 1.6 & 1400 & 4.7 & 1.0 \\
\hline 4383 & 3.5 & 0.6 & 1406 & 5.1 & 0.3 \\
\hline 4428 & 3.6 & 1.1 & 1445 & 5.2 & 0.4 \\
\hline 4458 & 3.3 & 0.9 & 1460 & 4.9 & 0.4 \\
\hline 4661 & 3.0 & 0.7 & 1461 & 4.7 & 0.6 \\
\hline 4907 & 2.8 & 1.0 & 1481 & 4.2 & 0.5 \\
\hline 5048 & 3.2 & 0.7 & 1563 & 5.7 & 1.1 \\
\hline 200613 & 4.9 & 0.5 & 1665 & 4.6 & 0.7 \\
\hline 202316 & 2.7 & 0.7 & 202063 & 6.3 & 0.6 \\
\hline \multicolumn{3}{|c|}{$\begin{array}{l}\overline{v_{\mathrm{rot}} \sin i_{\mathrm{TO}}}=3.6 \pm 0.3 \mathrm{~km} \mathrm{~s}^{-1} \\
\mathrm{rms}_{\mathrm{TO}}=0.9 \mathrm{~km} \mathrm{~s}^{-1}\end{array}$} & \multicolumn{3}{|c|}{$\begin{array}{l}\overline{v_{\mathrm{rot}} \sin i_{\mathrm{SG}}}=5.0 \pm 0.2 \mathrm{~km} \mathrm{~s}^{-1} \\
\mathrm{rms}_{\mathrm{SG}}=0.5 \mathrm{~km} \mathrm{~s}^{-1}\end{array}$} \\
\hline
\end{tabular}
and macro-turbulent and thermal velocity and instrumental profile.

larger than those due to other causes (e.g. macro-turbulence), the distribution would be much more scattered, given the random nature of the value of $\sin i$.

In fact, from simple statistical considerations it can be found that the scatter expected in case of a random distribution of the inclination of the rotational axis, $i$, assuming that all star rotate at the same rate, is $\sim 28 \%$ of the mean value of $v_{\text {rot }} \sin i$. We could also reverse the argument and infer a more stringent estimate of the upper limit of the typical rotation velocity from 
Table 13. Values for macro-turbulent velocity for which the $v_{\text {rot }}$ 's agree and corresponding values of the upper limits of the true $v_{\text {rot }}$ (see text).

\begin{tabular}{cccccccccccc}
\hline \hline & \multicolumn{3}{c}{ NGC 104-47 Tuc } & \multicolumn{3}{c}{ NGC 6397 } & \multicolumn{3}{c}{ NGC 6752 } & \multicolumn{3}{c}{ Mean value } \\
\hline & $\begin{array}{c}\zeta_{\text {RT,macro }} \\
\mathrm{km} \mathrm{s}^{-1}\end{array}$ & $\begin{array}{c}v_{\text {rot }} \\
\mathrm{km} \mathrm{s}^{-1}\end{array}$ & $\begin{array}{c}\sigma\left(v_{\text {rot }}\right) \\
\mathrm{km} \mathrm{s}^{-1}\end{array}$ & $\begin{array}{c}\zeta_{\text {RT,macro }} \\
\mathrm{km} \mathrm{s}^{-1}\end{array}$ & $\begin{array}{c}v_{\text {rot }} \\
\mathrm{km} \mathrm{s}^{-1}\end{array}$ & $\begin{array}{c}\sigma\left(v_{\text {rot }}\right) \\
\mathrm{km} \mathrm{s}^{-1}\end{array}$ & $\begin{array}{c}\zeta_{\text {RT,macro }} \\
\mathrm{km} \mathrm{s}^{-1}\end{array}$ & $\begin{array}{c}v_{\text {rot }} \\
\mathrm{km} \mathrm{s}^{-1}\end{array}$ & $\begin{array}{c}\sigma\left(v_{\text {rot }}\right) \\
\mathrm{km} \mathrm{s}^{-1}\end{array}$ & $\begin{array}{c}\bar{v}_{\text {rot }} \\
\mathrm{km} \mathrm{s}^{-1}\end{array}$ & $\begin{array}{c}\sigma\left(\bar{v}_{\text {rot }}\right) \\
\mathrm{km} \mathrm{s}^{-1}\end{array}$ \\
\hline TO & 5.9 & 4.2 & 1.1 & 6.6 & 2.9 & 0.8 & 5.9 & 4.0 & 1.0 & 3.5 & 0.4 \\
SG & 6.2 & 4.9 & 1.0 & 6.7 & 1.7 & 0.5 & 8.3 & 4.1 & 1.0 & 2.6 & 1.1 \\
\hline
\end{tabular}

the observed scatter of the $v_{\text {rot }} \sin i$ distribution. Under the reasonable assumption that all the stars of the same spectral type within the same cluster rotate at the same rate, we obtain then $\operatorname{rms}\left(v_{\text {rot }} \sin i\right)=v_{\text {rot }} \mathrm{rms}(\sin i)$, and $\overline{v_{\text {rot }} \sin i}=v_{\text {rot }} \overline{\sin i}$ therefore:

$v_{\text {rot }}=\frac{\operatorname{rms}\left(v_{\text {rot }} \sin i\right)}{\operatorname{rms}(\sin i)}$

and

$v_{\text {rot }}=\frac{\overline{v_{\text {rot }} \sin i}}{\overline{\sin i}}$.

The mean value and the scatter of the $\sin i$ function assuming a random inclination are easily calculated, and they result to be $\overline{\sin i}=\frac{\Pi}{4}$ and $\operatorname{rms}(\sin i)=\sqrt{\frac{2}{3}-\frac{\Pi^{2}}{16}}$; thus from Eqs. (6) and (7) we can obtain the values for the true rotational velocities for each of the groups of stars.

However, as it can be easily found using the results obtained so far for the mean and rms values of the $v_{\text {rot }} \sin i$ of the program clusters, such substitutions give different results. This is due to the fact that the adopted macro-turbulent velocity is an underestimate of the true one, therefore the mean values of $v_{\text {rot }} \sin i$ are overestimated whereas its rms is underestimated, given that the mean value and the rms are, respectively, decreasing and increasing functions of macro-turbulent velocity in the range considered (see Fig. 5).

Therefore, we computed iteratively the values of macroturbulent velocity for which the values of $v_{\text {rot }}$ obtained from Eqs. (6) and (7) agree at $0.1 \mathrm{~km} \mathrm{~s}^{-1}$ level. Figure 5 gives a graphical representation of this procedure. The dotted and dashed lines represent, respectively, the values of $v_{\text {rot }}$ as obtained from $\operatorname{rms}\left(v_{\text {rot }} \sin i\right)$ and from $\overline{v_{\text {rot }} \sin i}$. The vertical lines (which in the NGC 104-47 Tuc case coincide with the left edge of the panel) represent the values of macro-turbulent velocity for corresponding stars of Population I, i.e. the lower limits to the true value of the macro-turbulent velocity. The values for the macro-turbulent velocity for which the two values of $v_{\text {rot }}$ agree (i.e. the abscissa of the intersection of the dashed and dotted lines) are reported in Table 13. It is noteworthy that the mean values of the two determinations of $v_{\text {rot }}$ is almost constant over the range of macro-turbulent velocity values considered, and this suggests that it could provide good estimate of $v_{\text {rot }}$ despite the poor knowledge of the macro-turbulent velocity value. In order to obtain the error on the rotational velocities calculated with this procedure, we estimated the errors introduced for each of the groups of the program stars when assuming that the theoretical values of $\overline{\sin i}=\frac{\Pi}{4}$ and $\operatorname{rms}(\sin i)=\sqrt{\frac{2}{3}-\frac{\Pi^{2}}{16}}$ represent the true mean and rms of the

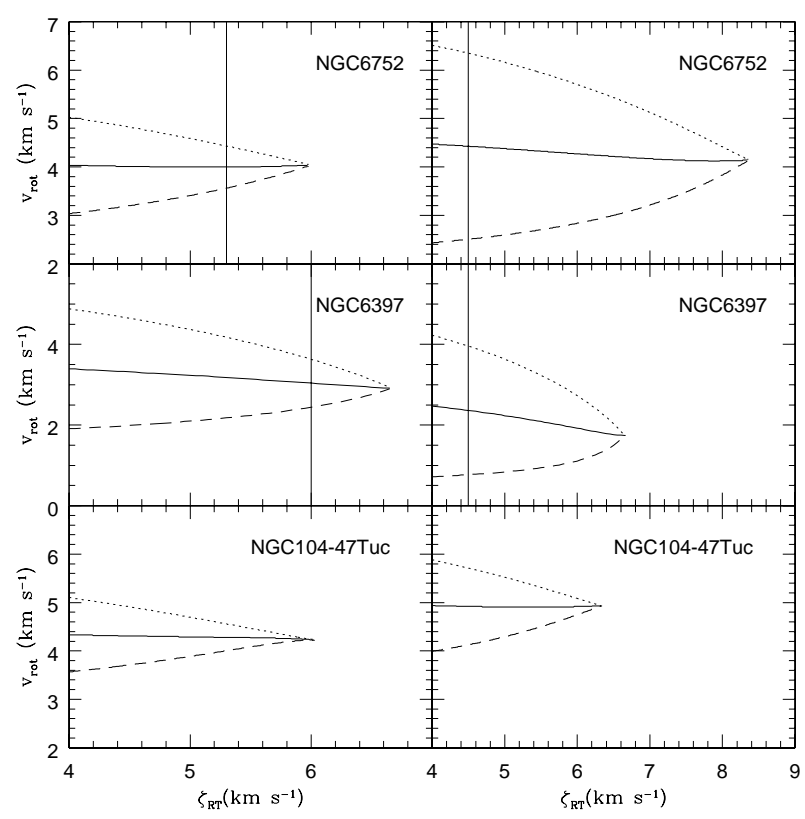

Fig. 5. Values of $v_{\text {rot }}$ from $\operatorname{rms}\left(v_{\text {rot }} \sin i\right)$ (dashed lines) and $\overline{v_{\text {rot }} \sin i}$ (dotted line). The panels on the left and right sides represent, respectively, the TO and SG stars. The solid lines represent the means of the two above mentioned values, which are remarkably constant over the range of macro-turbulent velocity values considered. The vertical lines mark the lower limit to the macro-turbulent velocities (see text). Such line in the case on NGC 104-47 Tuc is at $4 \mathrm{~km} \mathrm{~s}^{-1}$, at the edge of the plotted range.

$\sin i$ for a set of $N$ inclination $i$ values (where $N$ is the number of stars in each of the groups of the program stars). To this aim, we performed a Montecarlo simulation, which made an extraction of 100000 sets of $N$ random values of $i$, calculating for each of these sets the value of $\operatorname{rms}(\sin i)_{N}$ and $\overline{\sin }_{N}$. Thus, we built the distribution function of $\mathrm{rms}_{N}(\sin i)$ and $\overline{\sin }_{N}$, obtaining their $\sigma$ 's, which we used in the computation of the the error on the rotational velocities derived, respectively, from Eqs. (6) and (7).

It should be kept in mind that the values obtained in this way are not measurements of $\bar{v}_{\text {rot }}$, but upper limits. In fact, the assumption that all stars at the same evolutionary stage in a cluster have the same macro and micro-turbulent velocities and that they all rotate with the same true velocity is an approximation. Therefore, part of the scatters in our distributions is due to such differences in the parameters used and the scatters due only to the random orbital inclinations have a smaller true value. The scatter due to the differences in the atmospheric parameters is likely to be negligible with respect to that due to 
the random nature of the inclination, and we also expect that the distribution of the rotational velocities for the old population stars should have a small dispersion. Nevertheless, taking into account any non-constant distribution of such quantities would decrease the calculated values of $\bar{v}_{\text {rot }}$.

\section{Conclusions}

We have computed, using the cross-correlation technique, upper limits for the rotation of TO and SG stars in the globular clusters NGC 104, NGC 6397 and NGC 6752. Our analysis shows no evidence for large rotational velocities in any of the program stars. We can safely state that the stars under discussion, at least as far as the outer layers are concerned, rotate with low rotation velocity.

We measured the upper limit to the value of the projected rotation of the TO stars of $\overline{v_{\text {rot }} \sin i}=3.5 \pm 0.2 \mathrm{~km} \mathrm{~s}^{-1}$, and about $\overline{v_{\text {rot }} \sin i}=4.7 \pm 0.2 \mathrm{~km} \mathrm{~s}^{-1}$ for the SG. The errors do not take into account systematic uncertainties due to the assumption that the templates are identical, so a more realistic estimate of the error would probably be $\sim 1 \mathrm{~km} \mathrm{~s}^{-1}$ for both quantities.

Furthermore, if the observed dispersions of the values of $v_{\text {rot }} \sin i$ are compared with expectations based on random orientation of the rotational axis and on the assumption that all stars rotate at the same rate, we obtain an estimate of the upper limit values for the true mean rotational velocities, $\bar{v}_{\text {rot }} \leq 3.5 \pm 0.4 \mathrm{~km} \mathrm{~s}^{-1}$ and $\bar{v}_{\text {rot }} \leq 2.6 \pm 1.1 \mathrm{~km} \mathrm{~s}^{-1}$ respectively for TO and SG stars. These values are slightly larger than those expected for stars of the old population; however we have to keep in mind that these are upper limits and any scatter in the true rotational velocity distribution would decrease their value. However, they are small in comparison to the large rotational velocities measured in considerable fraction of Horizontal Branch stars; therefore the mechanisms responsible for the origin of the high values of the rotational velocities in such stars are still unclear. It could be explained by differential rotation within the star. If the star core rotates much faster than the outer layers, such rotation is undetectable during its main sequence phase, while, when the star reaches the Horizontal Branch, after shedding most of its envelope, the observation of the core rotation becomes possible (Sills \& Pinsennault 2000). Another possible explanation could be the acquisition of momentum during its evolution (e.g due to an asymmetric helium flash).
Acknowledgements. We wish to thank M. Centurion, L. Pasquini and P. Bonifacio, who performed the observations at UVES and E. Carretta for useful discussion. We are very grateful to P. Nissen for supplying data and informations. We also thank our anonymous referee for useful comments and suggestions.

\section{References}

Abt, H. A., \& Morrell, N. I. 1995, ApJS, 99, 135

Alonso, A., Arribas, S., \& Martínez-Roger, C. 1999, A\&AS, 140, 261

Asplund, M., Nissen, P. E., Primas, F., Lambert, D. L., \& Smith, V. V. 2003, in preparation

Balachandran, S. 1990 ApJ, 354, 310

Behr, B. B., Cohen, J. G., \& McCarthy, J. K. 2000a, ApJ, 531, 37

Behr, B. B., Djorgovski, S. G., Cohen, J. G., et al. 2000b, ApJ, 528, 849

Carney, B. W., Latham, D. W., Laird, J. B., \& Aguilar, L. A. 1994, AJ, 107,2240

Carretta, E., Gratton, R. G., Clementini, G., \& Fusi Pecci, F. 2000 ApJ, 533, 215

Carretta, E., et al. 2003, in preparation

de Medeiros, J. R., \& Mayor, M. 1999 A\&AS, 139, 433

Duflot, M., Figon, P., \& Meyssonnier, N. 1995, A\&AS, 114, 269

Evans, D. S. 1967 in Determination of radial velocities and their applications, ed. A. H. Batten, \& J. F. Heard (London: Academic press), IAU Symp., 30, 57

Gratton, R. G., Bonifacio, P., Bragaglia, A., et al. 2001, A\&A, 369, 87

Gray, D. F. 1992, The observation and analysis of stellar spectra, Cambridge Astrophys. Ser.

Gray, D. F. 1984, ApJ, 281, 719

Grenier, S. Baylac, M. O., Rolland , L., et al. 1999, A\&AS, 137, 451

Grundahl, F., VandenBerg, D. A, Stetson, P. B., Andersen, M. I., \& Briley, M. 2000, in The Galactic Halo: from Globular Clusters to field Stars, Proceedings of the 35th Liege International Astrophysics Colloquium, ed. A. Noels, P. Magain, \& D. Caro, Institut d'Astrophysique et de Geophysique, Liege, 503

Harris, W. E. 1996, AJ, 112, 1487

Latham, D. W., Tsevi, M., Carney, B. W., et al. 1998, AJ, 96, 576

Nissen, P. E., Primas, F., Asplund, M., \& Lambert, D. L. 2002, A\&A, 390,235

Peterson, R. 1983, ApJ, 275, 737

Sills, A., \& Pinsonneault, M. H. 2000, ApJ, 540, 489S

Skuljan, J., Hearnshaw, J. B., \& Cottrell, P. L. 2000, PASP, 112, 966

Soderblom, D. R., Pendleton, J., \& Pallavicini, R. 1989, AJ, 97, 539

Tonry, J., \& Davis, M. 1979, AJ, 84, 1511

Uesugi, A., \& Fukuda, I. 1982 Catalogue of stellar rotational velocities (revised), Departement of Astronomy, University of Kyoto

Wolff, S., \& Simon, T. 1997, PASP, 109, 759 